\title{
Nano-embedded microparticles based dry powder inhaler for lung cancer treatment
}

\author{
Nazimuddin CHISHTI 1 * (D), Mohamed Hassan DEHGHAN 2 (D) \\ 1 Department of Drug Regulatory Affairs, Wockhardt Research Centre, D-4, M.I.D.C., Chikalthana, Aurangabad \\ (M.S) 431006, India. \\ 2 Department of Pharmaceutics, Faculty of Y.B. Chavan College of Pharmacy, Aurangabad (MS) 431001, India. \\ * Corresponding Author. E-mail: nazimchishti@yahoo.com (N.C.); Tel. +91-989-087 3763.
}

Received: 16 December 2019 / Revised: 10 February 2020 / Accepted: 14 February 2020

\begin{abstract}
Lower efficacy of chemotherapeutic agents through the systemic route for treatment of lung cancer is attributed to its lower concentration in the lungs. Conversely, higher concentrations of drug in the lungs can be achieved by pulmonary administration via the inhalation route. For effective deposition of the formulation at the target region (small airways and alveoli) of the lung, the aerodynamic diameter has to be controlled (1-5 $\mu \mathrm{m})$ and its retention is of key importance. The present study attempted to design a dry powder inhalation formulation with combined benefits of micron- and nano-sized particles [nano-embedded microparticles (NEMs)], which upon redispersion, results in nanoparticles (NPs) exhibiting good retention in the lungs. The present attempt is the foremost one to utilize NEMs administered by pulmonary route for the treatment of lung cancer. Docetaxel (DTX) NPs was formulated using sonication solvent evaporation technique and characterized. Thereafter, DTX-NPs were embedded into microparticles using the spray drying technique. The NEMs exhibited the desired flow properties with Carr's index $10.18 \pm 2.79$ and Hausner ratio $1.11 \pm 0.034$. The mass median aerodynamic diameter was $3.74 \pm 0.11 \mu \mathrm{m}$ and the fine particle fraction $42.96 \pm 1.66 \%$. Redispersed NP fraction was $47.78 \pm 4.65 \%$ with NPs retaining the desired properties. NPs demonstrated a sustained release of upto $144 \mathrm{~h}$. The particle size and PDI of the redispersed NPs were unaffected. NEMs displayed stability upon charging under accelerated conditions for upto 3 months. Comparison of the cytotoxicity of DTX and DTX-NEMs revealed that the DTX-NEMs had more cytoxicity owing to the increased uptake of liberated NPs by cells. The prepared formulation could successfully entrap NPs (with mucus barrierevading properties) in lactose microparticles, which can be deposited in the lungs and eventually, disintegrate to give back NPs under simulated lung conditions. The results suggest that the developed NEMs can be used in inhaled chemotherapy for the treatment of non-small cell lung cancer.
\end{abstract}

KEYWORDS: Nano-embedded microparticles; docetaxel; dry powder inhaler; lung cancer; aerodynamic properties.

\section{INTRODUCTION}

The pulmonary drug delivery route has proven to be a prospective route for the local and systemic therapies following inhalation [1-5]. The delivery of nanoparticles via the inhalation route for therapeutic benefits has gained much attention in the recent years, driven by the advantages such as enhanced bioavailability, reduced frequency of dosing, allayed side effects and the capability of effectively evading the phagocytic and mucociliary clearance mechanisms of the lung after deposition in the desired region [1,5,6,79]. Utilization of nanoparticles in chemotherapy has improved the therapeutic efficacy in lung cancer to a considerable extent [10-13]. Successful delivery of particles to the anticipated region in the lungs via pulmonary route demands control of the theoretical aerodynamic diameter $(\mathrm{dAt})$ at $1-5 \mu \mathrm{m}$. Particles of $\mathrm{dAt}<1 \mu \mathrm{m}$ were breathed out because of low inertia, while those $>5 \mu \mathrm{m}$ were mainly deposited in the mouth and throat regions because of the potential impact. Effective delivery of the nanoparticles to the desired pulmonary area while retaining the benefits of nanoparticles is the key for desired effectiveness; this is highly dependent on the particle size of the nanoparticles [14-16]. This could be realised either with the use of NP aqueous suspensions delivered by nebulizers, formulation of micron-sized dry powders composed of $\mathrm{NP}$, or the Nano-embedded Microparticles (NEMs), also called as the Trojan particles, prepared by various techniques such as spray drying (SD), spray freeze drying (SFD) and electrostatic assembly [17].

The literature reports the use of sugars such as lactose, trehalose, or mannitol, as inert micron-sized carriers of NPs for use in the deposition of NPs in the lung (NEMs) [18,19-23]. The microparticles dissolve

How to cite this article: Chishti N, Dehghan MH. Nano-embedded microparticles based dry powder inhaler for lung cancer treatment. J Res Pharm. 2020; 24(3): 425-435. 
and dissociate to release the embedded NPs at the surface small airways and of alveoli. Spray drying process is a rapid, single-step process that is known to produce inhalable powder by converting an extremely small liquid droplets to a dry product of powder with the optimum micromeritics for pulmonary delivery $[14,20,24]$. The excipient matrix prevents irreversible agglomeration during the process of drying by the formation of a bridge among the nanoparticles. In this study, NPs that released drug in a sustained manner and have enhanced retention time (owing to their immune escape) were engineered into micron-sized dry powders by SD using lactose as the carrier, followed by characterization studies on NEMs. The NEMs are expected to get deposited in small airways and alveoli following inhalation [25-27]. The released nanoparticles are known to selectively deposit in the tumour tissues with limited deposition in the normal tissues and thus increase the effectiveness of anticancer agent such as docetaxel [28]. Docetaxel belongs to the taxane class of anticancer drugs and is used effectively against wide variety of cancers and approved by US-FDA for non-small cell lung cancer. Docetaxel exerts the cytotoxic effect by inhibiting microtubules function and disrupting the micro-tubular network. Docetaxel is practically insoluble in water and has a log $\mathrm{p}$ value of 4.10. Due to its water insolubility, an intravenous injection formulation was prepared with combination of ethanol and tween 80 in a commercial docetaxel formulation (Taxotere ${ }^{\circledR}$ ) to increase its solubility. Issues were identified with the said formulation like hypersensitivity reactions, decreased uptake by tumor tissue, and increased exposure of other body compartments to the drug. The root cause suspected was use of the ingredients ethanol and tween 80 . The presented study aimed to overcome these adverse events, increase the concentration of drug in lungs and at the same time reduce the unwanted exposure of other body organs to anticancer drug.

\section{RESULTS}

\subsection{Characterization of DTX-NPs}

\subsubsection{Particle size, PDI, Zeta potential and Entrapment efficiency}

DTX-NPs were prepared by emulsification-solvent evaporation methodology using PLGA as a biodegradable polymer and PLX-188 as a helper molecule. The particle size, zeta potential, entrapment efficiency and PDI of the formulated nanoparticles are shown in Table 1.

Table 1. Particle size, PDI, zeta potential and entrapment efficiency of formulated nanoparticles (NPs).

\begin{tabular}{ccccc}
\hline Formulation & $\begin{array}{c}\text { Particle size } \\
(\mathbf{n m})\end{array}$ & $\begin{array}{c}\text { Zeta potential } \\
\mathbf{( m V )}\end{array}$ & $\begin{array}{c}\text { Entrapment efficiency } \\
\mathbf{( \% )}\end{array}$ & $\begin{array}{c}\text { Polydispersity } \\
\text { index(PDI) }\end{array}$ \\
\hline DTX-NPs & 222.1 & -34.8 & 58.2 & 0.121 \\
\hline
\end{tabular}

\subsubsection{Morphology of nanoparticles}

From SEM and TEM images (Figure 1), it can be observed that the prepared nanoparticles of PLGA containing docetaxel with PLX-188 as a helper molecule had a smooth surface and were homogenous with no aggregation.

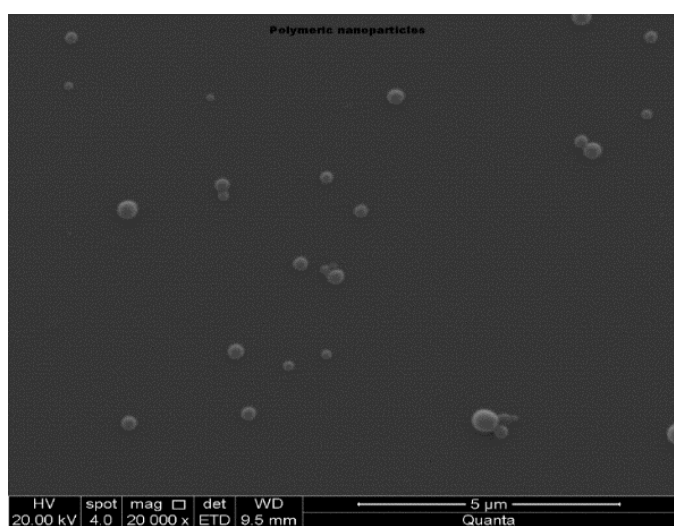

a

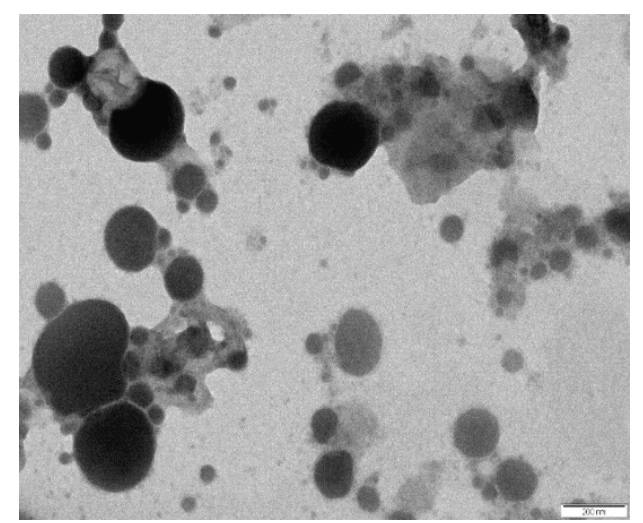

b

Figure 1. Morphology of nanoparticles a) Scanning electron microscope (SEM) image and b) Transmission electron microscope (TEM) image. 


\subsubsection{In vitro release of docetaxel from DTX-NPs}

The in vitro drug release profile (Figure 2) was studied for DTX-NPs formulation. The nanoparticles exhibited a biphasic drug release prototype and demonstrated a best fit with the Higuchi model (determination coefficient as $\mathrm{R}^{2}=0.91$ ).

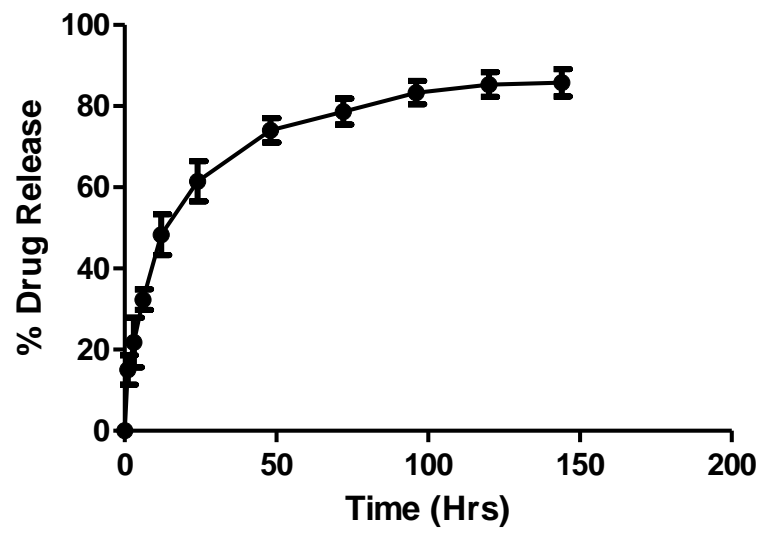

Figure 2. In vitro release profile of NPs.

\subsection{Characterization of DPI}

\subsubsection{Optimization of SD parameters}

The percentage yield, particle size and PDI obtained for NEMs produced by utilizing different SD inlet temperature are tabulated in Table 2.

Table 2. Correlation between inlet temperature and yield, particle size and PDI of NEMs.

\begin{tabular}{cccc}
\hline $\begin{array}{c}\text { Inlet temperature } \\
\left({ }^{\circ} \mathrm{C}\right) \text { [Actual/Set] }\end{array}$ & Yield $(\mathbf{\%})$ & Particle size $(\boldsymbol{\mu m})$ & $\begin{array}{c}\text { Polydispersity } \\
\text { index }(\text { PDI })\end{array}$ \\
\hline $80 / 80$ & 51 & 5.1 & 2.1 \\
$120 / 120$ & 69 & 3.2 & 0.7 \\
$160 / 160$ & 73 & 30 & 1.7 \\
\hline
\end{tabular}

\subsubsection{Nano embedded microparticle size, its morphology and flowability}

The DTX-NPs formulation was SD to obtain dry powder inhaler of size 2-5 $\mu \mathrm{m}$. Upon evaluating the particle size distribution, it was observed that the mean size was $<4 \mu \mathrm{m}$. The SEM image for the NEMs is as shown in Figure 3.

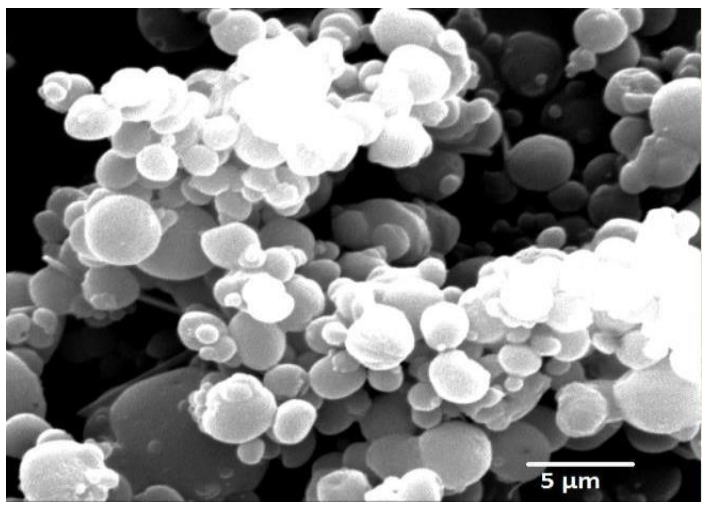

Figure 3. SEM image for nano-embedded microparticles.

The flowability of the powder was also studied using the Hausner ratio and CI. The results obtained are shown in Table 3. 
Table 3. Evaluation of flow properties of nano embedded microparticles (NEMs).

\begin{tabular}{ccccc}
\hline Formulation & $\begin{array}{c}\text { Bulk density } \\
(\mathrm{g} / \mathrm{ml})\end{array}$ & $\begin{array}{c}\text { Tapped density } \\
(\mathrm{g} / \mathrm{ml})\end{array}$ & $\begin{array}{c}\text { Carr's compressibility } \\
\text { index }\end{array}$ & Hausner ratio \\
\hline NEMs & $0.118 \pm 0.005$ & $0.132 \pm 0.010$ & $10.18 \pm 2.79$ & $1.11 \pm 0.034$ \\
\hline
\end{tabular}

\subsubsection{In vitro aerodynamic particle size distribution (Aerodynamic behavior)}

Aerosolization of the SD NEMs was evaluated in vitro by using the Next Generation Impactor. Size 3 hard gelatin capsules were filled with approximately $8 \mathrm{mg}$ of the SD powder and placed in a cyclohaler ${ }^{\circledR}$ (Cipla Ltd.; Mumbai). The capsule was punctured and its content was aerosolised by using vacuum pump (Erweka; Heusenstamm, Germany) with a gas flow of $60 \mathrm{~L} / \mathrm{min}$ for $4 \mathrm{~s}$. The powder concentrations in the induction port, NGI cups and pre-separator were estimated by rinsing with DMSO, followed by analysis by HPLC. MMAD (that is the diameter corresponding to the cumulative mass fraction of 50\% (probit of 5) and geometric standard deviation (GSD) were determined by feeding the cumulative powder mass fractions in CITDAC software. FPF was calculated as the sum of powder mass fraction from stage 3 to MOC, and it represents the fraction of powder with a diameter $<5 \mu \mathrm{m}$ (Table 4 ).

Table 4. Emitted dose, fine particle fraction, mass median aerodynamic diameter and geometric standard deviation for NEMs.

\begin{tabular}{lcccc}
\hline Formulation & ED (\%) & FPF (\%) & MMAD & GSD \\
\hline NEMs & $92.03 \pm 0.12$ & $42.96 \pm 1.66$ & $3.74 \pm 0.11$ & $1.87 \pm 0.05$ \\
\hline
\end{tabular}

\subsubsection{Redispersibility of NEMs into NPS}

Excess of simulated lung fluid under shaking was used for testing the redispersibility of NEMs. The supernatant was analysed by nanosizer to estimate the size and PDI of the redispersed nanoparticles. The weight of the lyophilised pellet was recorded and related to that of the non-redispersed fraction of the powder. The fraction of the redispersed nanoparticles was calculated by assuming that lactose was completely dissolved. The percentage of the redispersed powder and redispersed NP fraction yielded are shown in Table 5.

Table 5. Percent redispersed powder and redispersed NP fraction from NEMs.

\begin{tabular}{lcc}
\hline Formulation & Redispersed powder [\%] & Redispersed NP fraction [\%] \\
\hline NEMs & $97.78 \pm 0.30$ & $47.78 \pm 4.65$ \\
\hline
\end{tabular}

Approximately $47 \%$ of the nanoparticles were redispersed. Particle size and PDI were compared as shown in Table 6 and Figure 4 for freshly prepared NPs and redispersed NPs. Merely a slender increase in the size and PDI in comparison with those of the freshly prepared nanoparticles was observed for the samples SD powder with lactose.

\subsubsection{Moisture content}

Moisture content ascertains the stability of formulation on storage and affects the deaggregation upon inhalation. The moisture content $(\% \mathrm{w} / \mathrm{w})$ of the delivery systems was determined by Karl Fischer titration method. The moisture content of the SD powder of NEMs was $1.4 \pm 1.26 \%$.

\subsubsection{Stability data}

The SD powder (NEMs) was studied for its stability under accelerated stability conditions. At the testing interval, NEMs were analysed to reveal no aggregation or lump formation and that the product was stable. No noteworthy change was noted in description, content of entrapped drug, particle size and PDI in comparison to those measured at the initial time-points (Table 7).

Table 6. Comparison of Particle size and PDI of freshly prepared NPs and redispersed NPs.

\begin{tabular}{ccc}
\hline Formulation & Particle size $(\mathbf{n m})$ & PDI \\
\hline Freshly prepared NPs & $214.33 \pm 4.01$ & $0.123 \pm 0.014$ \\
\hline Redispersed NPs & $223 \pm 4.31$ & $0.432 \pm 0.031$ \\
\hline
\end{tabular}




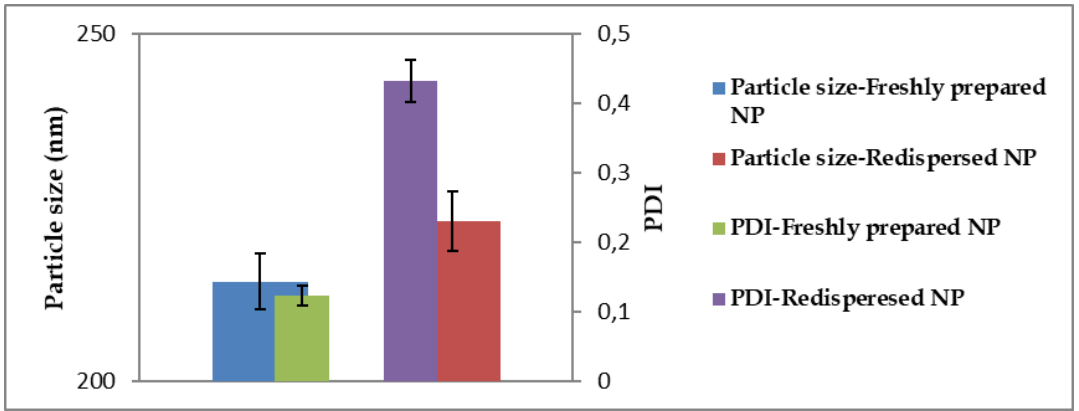

Figure 4. Comparison chart for Particle size and PDI of freshly prepared NPs and redispersed NPs.

Table 7. Accelerated stability data for NEMs.

\begin{tabular}{lccc}
\hline Parameters & Initial & 1 month & 3 months \\
\hline Physical description & White powder & White powder & White powder \\
Particle size & $3.74 \pm 0.06$ & $3.85 \pm 0.13$ & $3.86 \pm 0.32$ \\
PDI & $0.21 \pm 0.1$ & $0.25 \pm 0.03$ & $0.28 \pm 0.07$ \\
Drug content & $98.16 \pm 0.81$ & $96.83 \pm 1.87$ & $96.16 \pm 1.72$ \\
\hline
\end{tabular}

\subsubsection{In vitro cytotoxicity evaluation study}

In vitro cell cytotoxicity study was performed on A549 cells. The cell viability was determined after the treatment with plain docetaxel and DTX-NEMs at different concentrations after $48 \mathrm{~h}$. The statistics was run to define the $\mathrm{IC}_{50}$ value. One-way ANOVA followed by "Dunnett's Multiple Comparison Test" were used to perform the statistical comparison (Figure 5).

Cytotoxicity studies against $\mathrm{A549}$ cell line at $48 \mathrm{~h}$

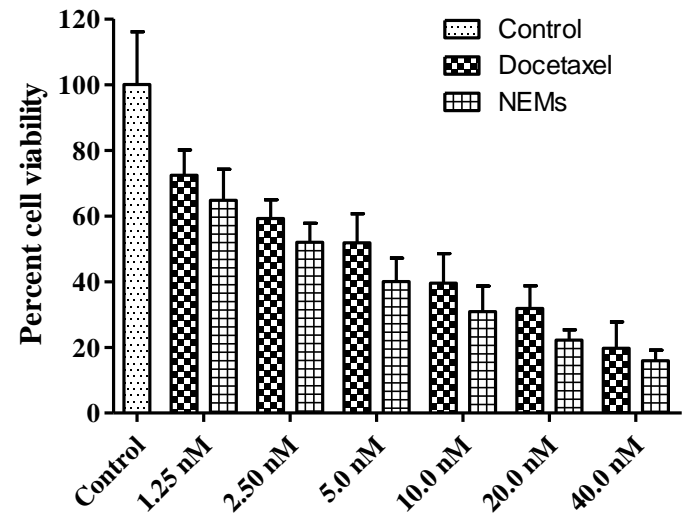

Figure 5. Cytotoxicity evaluation in A549 cell line

\section{DISCUSSION}

\subsection{Characterization of DTX-NPs}

Particle size of the nanoparticles was around $222 \mathrm{~nm}$, which is the desired size for penetration in the airway mucus. PDI of 0.121 indicated a uniform particle size distribution. The entrapment efficiency was around $58.2 \%$. Zeta potential of $-34.8 \mathrm{mV}$ demonstrated a stable nanoparticle suspension state before drying, which is attributable to PLX-188 as a non-ionic surfactant. As observed in the SEM and TEM studies, the NEMs exhibited a smooth surface and spherical shape. The in vitro release study revealed that the formulated NPs were capable of sustaining the release of docetaxel for up to $144 \mathrm{~h}$. An initial burst release was noted due to the drug adsorbed unto the surface of NPs and its subsequent release was a result of diffusion and matrix erosion of the polymer. Thus, NPs of the desired properties were formulated based on the optimised process established in our previous reported work. The NPs so prepared could be retained in 
the target region of the lung after deposition and evasion of the body's immune clearance due to their size and PLX-188 coating, as reported elsewhere [25,29].

\subsection{Characterization of DPI}

During the optimization of the SD parameters, it was observed that the yield, particle size and PDI were dependent on the inlet temperature. With the increase in the inlet temperature, yield increased and, similarly, the particle size and PDI also increased. Increase in the yield was explained by Maury et al. that owing to high residual moisture content in powder when low temperature is used during drying, the recovery of powder was reduced significantly [19]. The particle size of NEMs obtained using the inlet temperature of $80^{\circ} \mathrm{C}$ and $160^{\circ} \mathrm{C}$ was larger than that obtained using $120^{\circ} \mathrm{C}$. At a low temperature, larger particle size can be due to the cohesion of particles and agglomeration, while, at higher temperature, the particles stickiness after melting of some of the portion may lead to agglomeration. Similar outcomes have been reported in the literature [30]. The lowest PDI was obtained in formulation SD using inlet temperature of $120^{\circ} \mathrm{C}$ as compared to those at $80^{\circ} \mathrm{C}$ and $160^{\circ} \mathrm{C}$; this difference can be due adequate drying at $120^{\circ} \mathrm{C}$ because of which agglomeration is prevented and the particles formed are uniform, small in size and spherical in shape.

The particle size of NEMs was observed to be in the desired range of 3-5 $\mu \mathrm{m}$, which is necessary for deposition in small airways and deep lung. The SEM image indicated a regular and spherical shape with smooth surfaces for the NEMs. To ensure that the formulated powder has a suitable flowability, the Hausner ratio and CI were measured. The Hausner ratio and Carr's compressibility index indicated that the prepared formulations have the desirable flowability. As per European Pharmacopoeia, a Hausner ratio $>1.25$ usually indicates poor flowability. In case of $\mathrm{CI}$, the smaller the value better is the flow property. In general, powders with a CI value $>25$ exhibit poor flowability as recorded in European Pharmacopoeia. Similar results have been reported in the literature [2]. The aerodynamic properties of NEMs confirmed that the developed dry powder has the expected flowability and that approximately $92 \%$ of the capsule content is emitted after actuation. The size of particles affects both the site of deposition and the mass of inhaled drug that deposits in the respiratory tract. For the developed formulation, based on the in vitro aerodynamic particle size distribution, it can be observed that MMAD was achieved in the required range (3.63 to 3.85) for deposition in small airways and deep lung based on the size, shape and density of particles. The respirable or FPF was observed to be around $42.96 \%$. GSD $>1.5$ explained the polydispersed nature of powder which is common in pharmaceutical aerosol preparation. Once deposited in the lungs, microparticles are anticipated to disintegrate and release the embedded nanoparticles. For successful disintegration of micro-carriers, it is expected that there should not be any irreversible aggregation of NPs while they are SD to form NEMs. With the formulation parameters selected in this method, that is the concentration of lactose, NPs content in feed suspension and the optimised SD parameters produced NEMs with good percentage of redispersed powder and NP fraction. There was only a slight increase in the particle size and PDI of redispersed NPs and freshly prepared ones. The results indicate no irreversible aggregation of NPs. Moisture content of nearly $1.4 \%$ indicates that the conditions optimised for the development of SD powder were best suited for the removal of moisture content from the prepared powder. The prepared NEMs demonstrated stability upon charging at accelerated stability conditions for up to 3 months. Upon comparison of cytotoxicity of docetaxel and docetaxel-NEMs, docetaxel-NEMs was found to have more cytoxicity owing to an increased uptake of NPs by cells released from NEMs.

\section{CONCLUSION}

In the present study, we formulated docetaxel nanoparticles with PLGA as a polymer and PLX-188 as a helper molecule displaying optimal physicochemical properties in terms of the size, uniformity, loading efficiency and in vitro release. The DTX-NPs (size approximately $200 \mathrm{~nm}$ ) were readily SD and converted into microparticles (of size range 1-5 $\mu \mathrm{m}$ ) that exhibited suitable flowability and aerodynamic behaviour. DTX-NEMs DPI formulation showed great potential for use in the localised delivery of DTX molecules to the lung, for passively targeting non-small cell lung cancer (NSCLC) and as an imperative element of combination therapy along with the systemic or local treatment of lung cancer. In short, it was envisioned that this study may have implications in the development of novel DPIs with the desired fine particle dose disintegrating into NPs, which can evade the physiological barriers and improve the outcome of lung cancer therapy. 


\section{MATERIALS AND METHODS}

Docetaxel was a kind gift sample from Glenmark Pharmaceuticals (Mumbai, India). PLGA (lactide:glycolide ratio of 75:25- Resomer 752H) was sorted from Evonik Industries (Mumbai, India), Poloxamer 188 (PLX-188) and lactose monohydrate was purchased from BASF, (Mumbai, India) and Sigma Aldrich (Mumbai, India), respectively. Organic solvents of analytical reagent grade (acetonitrile and ethyl acetate) were acquired from Fisher Scientific (Mumbai).

\subsection{Formulation of NEMs}

PLX-188-coated PLGA-nanoparticles were prepared by emulsification-solvent evaporation method and SD with lactose solution to get NEMs [25]. Concisely, docetaxel $(10 \mathrm{mg})$ and PLGA $(200 \mathrm{mg})$ were solubilised in ethyl acetate $(4 \mathrm{~mL})$. The resulting solution (internal phase) was then added slowly to $4 \mathrm{~mL}$ of $0.3 \% \mathrm{w} / \mathrm{v}$ aqueous surfactant solution (external phase) under constant vortexing. On complete addition of the oil phase to the aqueous phase, the resultant phase system was subjected to continual vortexing for $15 \mathrm{~s}$ and, thereafter, probe sonicated without delay ( $60 \%$ of amplitude and time $45 \mathrm{~s}$ ). The resultant nanoparticle suspension was subsequently transferred to the external phase under stirring condition for the hardening of nanoparticles. Later, the organic solvent was evaporated overnight. Centrifugation $\left(17,000 \mathrm{rcf}, 4^{\circ} \mathrm{C}, 15 \mathrm{~min}\right)$ (Kubota 6500; China) was then performed to collect the nanoparticles from the pellet. The supernatant was carefully discarded to avoid any disorder of the pellet state and rinsed thrice to eliminate un-entrapped docetaxel. The pellet was then re-dispersed in deionised water using a bath sonicator and the final volume was made up to $5 \mathrm{~mL}$. Thus, the formed nanoparticles were suspended in $0.5 \% \mathrm{w} / \mathrm{v}$ lactose solution and spray dried (SD) using a spray dryer (Labultima; Mumbai, India). The NP concentration in the feed was maintained at $2.5 \mathrm{mg} / \mathrm{mL}$. The homogeneous dispersion was atomised using a $0.5-\mathrm{mm}$ nozzle. The optimised inlet temperature was $120^{\circ} \mathrm{C}$, the feed flow rate was $2 \mathrm{~mL} / \mathrm{min}$ and the resultant outlet temperature was $70^{\circ} \mathrm{C}$. The SD powder was collected and stored in a dessicator under the vacuum at the room temperature until further use.

\subsection{Characterization of nanoparticles}

\subsubsection{Particles size, polydispersity index (PDI) and zeta potential}

The mean particle size, zeta potential and PDI of poloxamer-coated PLGA nanoparticles containing docetaxel (DTX-NPs) were analysed by means of the Malvern Zeta Sizer Instrument (Malvern; Worcestershire, UK). The pellet obtained after centrifugation was re-suspended in deionised water for further analysis.

\subsubsection{Entrapment efficiency}

The assessment of the percentage drug entrapped in DTX-NPs was performed by the HPLC method. Briefly, the supernatant obtained after centrifugation was discarded, and the pellets (sediment) were dissolved in acetonitrile. The suspension was analysed on HPLC (Shimadzu; Kyoto, Japan) equipped with SPD-M20A diode array detector and Luna C18 column (Phenomenex Inc., USA). The mobile phase (acetonitrile: orthophosphoric acid $=58: 42$ ) was pumped at a flow rate of $1.0 \mathrm{~mL} / \mathrm{min}$, constant column temperature of $37^{\circ} \mathrm{C}$ and injection volume of $10 \mu \mathrm{L}$. Docetaxel eluted at $7.4 \min$ at $\lambda$-max of $230 \mathrm{~nm}$.

\subsubsection{Morphology of nanoparticles}

DTX-NPs were suspended in deionised water to study their surface texture. Scanning electron microscopy (SEM) Field Electron \& Ion Company (FEI), Quanta 200 (FEI, Hillsboro, USA), accelerating voltage $(1.0 \mathrm{kv})$ with high vacuum was used for this purpose. Transmission electron microscopy (TEM) (Philips; Hillsboro, USA; CM200, 20-200kv, 2.4 Angstrom resolution) was used to study the texture of DTXNPs. Nanoparticle suspension was taken onto carbon-coated copper grids and dried completely. Next, $2 \%$ $\mathrm{w} / \mathrm{v}$ uranyl acetate was used for staining the sample, followed by capturing the images.

\subsubsection{In vitro release of docetaxel}

The DTX-NPs formulation prepared was studied for its in vitro release pattern. Polysorbate- $80(0.5 \%)$ as surfactant in phosphate-buffered saline (PBS; pH 7.4) was used as the release media while maintaining the sink conditions. DTX-NPs were suspended in $10-\mathrm{mL}$ release media in polypropylene tubes. The assembly was placed in a constant temperature $\left(37^{\circ} \mathrm{C}\right)$ water bath under magnetic stirring at $350 \mathrm{rpm}$. Aliquots were removed from tubes and analysed at defined intervals by HPLC. 


\subsection{Characterization of DPI}

\subsubsection{Optimization of spray drying (SD) parameters}

A study was designed to optimise the SD parameters for obtaining NEMs of the desired quality. For this purpose, formulations with fixed feed concentration were $\mathrm{SD}$ by using different inlet temperatures, such as $80^{\circ} \mathrm{C}, 120^{\circ} \mathrm{C}$ and $160^{\circ} \mathrm{C}$. Parameters such as the yield percentage, particle size and PDI were estimated for the NEMs produced from the formulations.

\subsubsection{Scanning electron microscopy}

SEM of the SD powder was undertaken to study the morphological nature of the microparticles (e.g. the spherical shape and aggregation). SEM, FEI, Quanta 200 (FEI, Hillsboro, USA), at the accelerating voltage of $1.0 \mathrm{kv}$ with high vacuum was used.

\subsubsection{Flowability properties}

Hausner ratio and Carr's index $(\mathrm{CI})$ were determined to assess the flowability of the test powder. The $\mathrm{CI}$ values helped in computing the tendency of the powder to consolidate. The rearrangement in the packing of the micro-particular powder bed due to the tapping procedure was expressed as the CI. Briefly, $2 \mathrm{~g}$ of the sample was subjected to 100 taps in a Microprocessor Tap (density tester-1951; Electronics India), and the CI was calculated from the tapped and bulk density of the SD powders using the Equation 1 given below:

$C I=\left(1-\frac{\rho_{B}}{\rho_{T}}\right) \times 100$

Where, $\rho_{T}$ is tapped bulk density and $\rho_{B}$ is freely bulk density of powder.

Hausner ratio was calculated using the following Equation 2:

$$
\text { Hausner ratio }=\frac{\rho_{T}}{\rho_{B}}
$$

\subsubsection{In vitro aerodynamic particle size distribution of dry powder (characterization of aerodynamic properties)}

In vitro aerodynamic particle size distribution of DPI or the aerodynamic performance was evaluated using the Next-Generation Impactor (NGI) 170 (MSP Corporation, USA). The total quantity of $8 \mathrm{mg}$ of NEMs was loaded into each of 30 hard gelatin capsules (size 3). Accordingly, a pressure of $4 \mathrm{kPa}$ and an air stream of $60 \mathrm{~L} / \mathrm{min}$ were created throughout the system by appending the NGI outlet to a vacuum pump for $4 \mathrm{~s}$. The powder deposited in stages 1-7 and micro-orifice collector (MOC), the mouthpiece and the pre-separator device were collected by dimethyl sulfoxide (DMSO) rinsing. The drug deposition data thus obtained was used to calculate the percentage of emitted dose (ED), the fine particle fraction (FPF), the mass median aerodynamic diameter (MMAD) and the geometric standard deviation (GSD). The FPF and ED were calculated as follows using the Equations 3 and 4, respectively:

$$
\begin{array}{ll}
F P F=\frac{F P D}{\text { Initial particle mass in capsule }} \mathrm{X} 100 & \text { [Eq. 3] } \\
E D=\frac{\text { Initial particle mass in capsule-Final mass remaining in capsule }}{\text { Initial particle mass in capsule }} \mathrm{X} 100 & \text { [Eq. 4] }
\end{array}
$$

\subsubsection{Moisture content}

The sample (approximately $15 \mathrm{mg}$ ) was weighed and subjected to Karl Fisher volumetric titration (Spectra Lab Instruments Pvt., Ltd.; Mumbai, India) to detect the moisture content of the dry powders.

\subsubsection{Recovery of nanoparticles from NEMs}

To evaluate the recovery of NPs from NEMs, $30 \mathrm{mg}$ of the SD powder of NEMs was weighed and placed in a pre-weighed Eppendorf tube. To these tubes, $6 \mathrm{~mL}$ of simulated lung fluid (SLF) was added, and 
the assembly was placed on a horizontal shaker with gentle shaking at $150 \mathrm{rpm}$ and temperature of $37^{\circ} \mathrm{C}$. The dispersion was centrifuged at $2000 \mathrm{~g}$ for $10 \mathrm{~min}$, and a small amount of the supernatant was withdrawn and analysed for particle size and PDI of NPs by Malvern Zeta Sizer Instrument. To the remaining pellet and liquid in tube, water was added and centrifugation was repeated at $2000 \mathrm{~g}$ for $10 \mathrm{~min}$, after which the supernatant was discarded. This washing step with water followed by centrifugation and discarding the supernatant was repeated thrice. Subsequent to the last washing cycle, the pellet was frozen to $-80^{\circ} \mathrm{C}$ before lyophilization (Christ, Osterode, Germany). The weight calculation for pellets was performed by subtracting the weight of the Eppendorf tube with that of the lyophilised pellet and the weight of the empty Eppendorf tube. Redispersed fraction of powder was computed by subtracting the pellet weight from the total mass of the powder and dividing it by the total powder mass. Pellet was believed to be only the non-dispersible microparticles or portion of it since lactose would completely dissolve. The redispersed fraction of the nanoparticles was measured by determining the relative ratio of the nanoparticles in the supernatant (difference between the amount of redispersed powder and lactose mass) to the theoretical nanoparticle content [20].

\subsubsection{Stability studies}

SD powder of NEMs was subjected to accelerated stability conditions (storage condition $2-8^{\circ} \mathrm{C}$ ) in order to scrutinise the stability upon storage. SD formulation was filled in gelatin capsule and packaged in HDPE bottles, followed by charging on stability for a 3-month period at a temperature of $25 \pm 2{ }^{\circ} \mathrm{C} / 60 \pm$ $5 \% \mathrm{RH}$. The stability parameters were the physical description of powder, drug content, particle size and PDI.

\subsubsection{In vitro cytotoxicity evaluation}

Lung cancer cell lines (A549 were procured from National Centre for Cell Science, Pune, India) were taken with Dulbecco's modified Eagle's medium (DMEM) into 96-well micro-titre plate at $1 \times 10^{4}$ cells/well density. In order to allow for attachment, the plate was incubated for $24 \mathrm{~h}$ at $37^{\circ} \mathrm{C}$ under $95 \%$ humidity with $\mathrm{CO}_{2}$ environment. Haemocytometer was used to determine the cell count. DMEM was then replaced with fresh medium $(0.2 \mathrm{~mL})$ containing different concentrations of free docetaxel and DTX-NEM set dose equivalent to $40,20,10,5,2.5$ and $1.25 \mathrm{nM}$ and incubated at $37^{\circ} \mathrm{C}$ for $48 \mathrm{~h}$. Medium from each well was replaced with $20 \mu \mathrm{L}$ of methyl thiazolyltetrazolium (MTT) solution, followed by incubation at $37^{\circ} \mathrm{C}$ for $4 \mathrm{~h}$. To each of the plate well, $100 \mu \mathrm{L}$ DMSO was added and the plate was vortexed for $15 \mathrm{~min}$ to dissolve the formazan crystals. ELISA reader at $570 \mathrm{~nm}$ filter was used to note the absorbance, and thus the percentage of viable cells was calculated. Graph pad prism (version 7.0) was used to plot the human lung cancer cell line (A549) experiment results for the estimation of $\mathrm{IC}_{50}$ value of DTX-NEMs. The $\mathrm{IC}_{50}$ value of DTX-NEMs was expressed as mean \pm SD.

Acknowledgements: Authors are thankful to Glenmark Pharmaceuticals (Mumbai, India) and Evonik Industries (Mumbai, India) for providing gift samples of docetaxel and Resomer $752 \mathrm{H}$ respectively.

Author contributions: Concept -C.N., D.MH.; Design - C.N., D.MH.; Supervision -D.MH.; Resources -C.N.; Materials -C.N.; Data Collection and/or Processing - C.N., Analysis and/or Interpretation -C.N.,D.MH.; Literature Search - C.N.; Writing - C.N.; Critical Reviews - C.N., D.MH.

Conflict of interest statement: The authors declared no conflict of interest in the manuscript.

\section{REFERENCES}

[1] Kaminskas LM, McLeod VM, Ryan GM, Kelly BD, Haynes JM, Williamson M, et al. Pulmonary administration of a doxorubicin-conjugated dendrimer enhances drug exposure to lung metastases and improves cancer therapy. J Control Release Off J Control Release Soc. 2014; 183: 18-26. [CrossRef]

[2] Bakhtiary Z, Barar J, Aghanejad A, Saei AA, Nemati E, Ezzati Nazhad Dolatabadi J, et al. Microparticles containing erlotinib-loaded solid lipid nanoparticles for treatment of non-small cell lung cancer. Drug DevInd Pharm. 2017; 43(8): 1244-1253. [CrossRef]

[3] Pallagi E, Karimi K, Ambrus R, Szabo-Revesz P, Csoka I. New aspects of developing a dry powder inhalation formulation applying the quality-by-design approach. Int J Pharm. 2016; 511(1):151-160. [CrossRef] 
[4] Bhardwaj A, Mehta S, Yadav S, Singh SK, Grobler A, Goyal AK, et al. Pulmonary delivery of antitubercular drugs using spray-dried lipid-polymer hybrid nanoparticles. Artificial Cells Nanomedicine Biotechnology. 2016; 44(6): 1544-1555. [CrossRef]

[5] Lee WH, Loo CY, Traini D, Young PM. Inhalation of nanoparticle-based drug for lung cancer treatment: Advantages and challenges.Spec Issue Inhal. 2015; 10(6): 481-489. [CrossRef]

[6] Zarogoulidis P, Chatzaki E, Porpodis K, Domvri K, Hohenforst-Schmidt W, Goldberg EP, et al. Inhaled chemotherapy in lung cancer: future concept of nanomedicine. Int J Nanomedicine. 2012; 7: 1551-1572. [CrossRef]

[7] Beck Broichsitter M, Kleimann P, Schmehl T, Betz T, Bakowsky U, Kissel T, et al. Impact of lyoprotectants for the stabilization of biodegradable nanoparticles on the performance of air-jet, ultrasonic, and vibrating-mesh nebulizers. Eur J Pharm Biopharm Off J Arbeitsgemeinschaft Pharm Verfahrenstechnik EV. 2012; 82(2): $272-280$. [CrossRef]

[8] Azarmi S, Tao X, Chen H, Wang Z, Finlay WH, Lobenberg R, et al. Formulation and cytotoxicity of doxorubicin nanoparticles carried by dry powder aerosol particles. Int J Pharm. 2006; 319(1-2): 155-161. [CrossRef]

[9] Roa WH, Azarmi S, Al-Hallak MHDK, Finlay WH, Magliocco AM, Lobenberg R. Inhalable nanoparticles, a noninvasive approach to treat lung cancer in a mouse model. J Control Release Off J Control Release Soc. 2011; 150(1): 49-55. [CrossRef]

[10] Tian J, Min Y, Rodgers Z, Wan X, Qiu H, Mi Y, et al. Nanoparticle delivery of chemotherapy combination regimen improves the therapeutic efficacy in mouse models of lung cancer. Nanomedicine Nanotechnol Biol Med. 2017; 13(3): 1301-1307. [CrossRef]

[11] Patel AR, Chougule MB, I T, Patlolla R, Wang G, Singh M. Efficacy of aerosolized celecoxib encapsulated nanostructured lipid carrier in non-small cell lung cancer in combination with docetaxel. Pharm Res. 2013; 30(5): 1435-1446. [CrossRef]

[12] Chen $\mathrm{R}, \mathrm{Xu} \mathrm{L}$, Fan $\mathrm{Q}$, Li M, Wang J, Wu L, et al. Hierarchical pulmonary target nanoparticles via inhaled administration for anticancer drug delivery. Drug Deliv. 2017; 24(1): 1191-1203. [CrossRef]

[13] Choi SH, Byeon HJ, Choi JS, Thao L, Kim I, Lee ES, et al. Inhalable self-assembled albumin nanoparticles for treating drug-resistant lung cancer. J Control Release Off J Control Release Soc. 2015; 197: 199-207. [CrossRef]

[14] Varshosaz J, Taymouri S, Hamishehkar H, Vatankhah R, Yaghubi S. Development of dry powder inhaler containing tadalafil-loaded PLGA nanoparticles. Res Pharm Sci. 2017; 12(3): 222-232. [CrossRef]

[15] Ryan GM, Kaminskas LM, Kelly BD, Owen DJ, McIntosh MP, Porter CJH. Pulmonary administration of PEGylatedpolylysine dendrimers: absorption from the lung versus retention within the lung is highly sizedependent. Mol Pharm. 2013; 10(8): 2986-2995. [CrossRef]

[16] Kuzmov A, Minko T. Nanotechnology approaches for inhalation treatment of lung diseases. J Control Release Off J Control Release Soc. 2015; 219: 500-518. [CrossRef]

[17] Yang Y, Cheow WS, Hadinoto K. Dry powder inhaler formulation of lipid-polymer hybrid nanoparticles via electrostatically-driven nanoparticle assembly onto microscale carrier particles. Int J Pharm. 2012; 434(1-2): 49-58. [CrossRef]

[18] Ogain ON, Li J, Tajber L, Corrigan OI, Healy AM. Particle engineering of materials for oral inhalation by dry powder inhalers. I-Particles of sugar excipients (trehalose and raffinose) for protein delivery. Int J Pharm. 2011 Feb 28; 405(1-2): 23-35. [CrossRef]

[19] Maury M, Murphy K, Kumar S, Shi L, Lee G. Effects of process variables on the powder yield of spray-dried trehalose on a laboratory spray-dryer. Eur J Pharm Biopharm Off J Arbeitsgemeinschaft Pharm Verfahrenstechnik EV. 2005; 59(3): 565-573. [CrossRef]

[20] Torge A, Grutzmacher P, Mucklich F, Schneider M. The influence of mannitol on morphology and disintegration of spray-dried nano-embedded microparticles. Eur J Pharm Sci Off J Eur Fed Pharm Sci. 2017; 104: 171-179. [CrossRef]

[21] Ungaro F, d'Angelo I, Coletta C, d'Emmanuele di Villa Bianca R, Sorrentino R, Perfetto B, et al. Dry powders based on PLGA nanoparticles for pulmonary delivery of antibiotics: modulation of encapsulation efficiency, release rate and lung deposition pattern by hydrophilic polymers. J Control Release Off J Control Release Soc. 2012; 157(1): 149_ 159. [CrossRef]

[22] Muralidharan P, Malapit M, Mallory E, Hayes DJ, Mansour HM. Inhalable nanoparticulate powders for respiratory delivery. Nanomedicine Nanotechnol Biol Med. 2015; 11(5): 1189-1199. [CrossRef] 
[23] Pilcer G, Amighi K. Formulation strategy and use of excipients in pulmonary drug delivery. Int J Pharm. 2010; 392(1-2): 1-19. [CrossRef]

[24] Ungaro F, d'Angelo I, Miro A, La Rotonda MI, Quaglia F. Engineered PLGA nano- and micro-carriers for pulmonary delivery: challenges and promises. J Pharm Pharmacol. 2012; 64(9): 1217-1235. [CrossRef]

[25] Chishti N, Jagwani S, Dhamecha D, Jalalpure S, Dehghan MH. Preparation, Optimization, and In Vivo Evaluation of Nanoparticle-Based Formulation for Pulmonary Delivery of Anticancer Drug. Med Kaunas Lith. 2019; 55(6): 294. [CrossRef]

[26] Maeda H. The enhanced permeability and retention (EPR) effect in tumor vasculature: the key role of tumorselective macromolecular drug targeting. Adv Enzyme Regul. 2001; 41: 189-207. [CrossRef]

[27] Zhang J, Wu L, Chan H-K, Watanabe W. Formation, characterization, and fate of inhaled drug nanoparticles. Adv Drug Deliv Rev. 2011; 63(6): 441-455. [CrossRef]

[28] Godugu C, Patel AR, Doddapaneni R, Marepally S, Jackson T, Singh M. Inhalation delivery of Telmisartan enhances intratumoral distribution of nanoparticles in lung cancer models. J Control Release Off J Control Release Soc. 2013; 172(1): 86-95. [CrossRef]

[29] Jain D, Athawale R, Bajaj A, Shrikhande S, Goel PN, Gude RP. Studies on stabilization mechanism and stealth effect of poloxamer 188 onto PLGA nanoparticles. Colloids Surf B Biointerfaces. 2013; 109: 59-67. [CrossRef]

[30] Broadhead J, Rouan SK, Hau I, Rhodes CT. The effect of process and formulation variables on the properties of spray-dried beta-galactosidase. J Pharm Pharmacol. 1994; 46(6): 458-467. [CrossRef]

This is an open access article which is publicly available on our journal's website under Institutional Repository at http://dspace.marmara.edu.tr. 